\title{
Nonadiabatic superconductivity in a Li-intercalated hexagonal boron nitride bilayer
}

\author{
Kamila A. Szewczyk ${ }^{*}$, Izabela A. Domagalska², Artur P. Durajski ${ }^{3}$ \\ and Radosław Szczęśniak ${ }^{1,3}$
}

\author{
Full Research Paper \\ Address: \\ ${ }^{1}$ Division of Theoretical Physics, Jan Długosz University in \\ Częstochowa, Ave. Armii Krajowej 13/15, 42-200 Częstochowa, \\ Poland, ${ }^{2}$ Quantum Optics and Engineering Division, Faculty of \\ Physics and Astronomy, University of Zielona Góra, Prof. Z. Szafrana \\ 4a, 65-516 Zielona Góra, Poland and ${ }^{3}$ Division of Physics, \\ Częstochowa University of Technology, Ave. Armii Krajowej 19, \\ 42-200 Częstochowa, Poland \\ Email: \\ Kamila A. Szewczyk ${ }^{*}$ - kamila.szewczyk@ujd.edu.pl \\ * Corresponding author \\ Keywords: \\ critical temperature; electron-phonon interaction; Li-hBN bilayer; \\ Li-intercalated hexagonal boron nitride ( $\mathrm{Li}-\mathrm{hBN})$; nonadiabatic \\ superconductivity; vertex corrections \\ Beilstein J. Nanotechnol. 2020, 11, 1178-1189. \\ doi:10.3762/bjnano.11.102 \\ Received: 23 March 2020 \\ Accepted: 08 July 2020 \\ Published: 07 August 2020 \\ Associate Editor: J. M. van Ruitenbeek \\ (C) 2020 Szewczyk et al.; licensee Beilstein-Institut. \\ License and terms: see end of document.
}

\begin{abstract}
When considering a Li-intercalated hexagonal boron nitride bilayer $(\mathrm{Li}-\mathrm{hBN})$, the vertex corrections of electron-phonon interaction cannot be omitted. This is evidenced by the very high value of the ratio $\lambda \omega_{\mathrm{D}} / \varepsilon_{\mathrm{F}} \approx 0.46$, where $\lambda$ is the electron-phonon coupling constant, $\omega_{\mathrm{D}}$ is the Debye frequency, and $\varepsilon_{\mathrm{F}}$ represents the Fermi energy. Due to nonadiabatic effects, the phonon-induced superconducting state in $\mathrm{Li}-\mathrm{hBN}$ is characterized by much lower values of the critical temperature $\left(T^{\mathrm{LOVC}}{ }_{\mathrm{C}} \in\{19.1,15.5,11.8\} \mathrm{K}\right.$, for $\mu^{*} \in\{0.1,0.14,0.2\}$, respectively) than would result from calculations not taking this effect into account $\left(T^{\mathrm{ME}} \in\{31.9,26.9\right.$, $21\} \mathrm{K})$. From the technological point of view, the low value of $T_{\mathrm{C}}$ limits the possible applications of Li-hBN. The calculations were carried out under the classic Migdal-Eliashberg formalism (ME) and the Eliashberg theory with lowest-order vertex corrections (LOVC). We show that the vertex corrections of higher order $\left(\lambda^{3}\right)$ lower the value of $T^{\mathrm{LOVC}}$ by a few percent.
\end{abstract}

\section{Introduction}

Low-dimensional systems such as graphene [1-5], silicene [6], borophene [7,8], and phosphorene [9-11] are mechanically stable only when placed on a substrate [12-14]. The substrate should be selected so that it changes the physical properties of the low-dimensional system as little as possible. In the case of graphene, the following substrate materials were used: Co [15], $\mathrm{Ni}$ [16-19], $\mathrm{Ru}$ [20,21], Pt [22,23], $\mathrm{SiC}$ [24-26], and $\mathrm{SiO}_{2}$ [2729]. Unfortunately, the obtained experimental data showed that 
the incompatible crystalline structure of the above materials leads to significant suppression of the carrier mobility of graphene $[13,30]$.

It is now assumed that the best substrate for graphene is hexagonal boron nitride $(\mathrm{hBN})$ with a honeycomb crystal structure in which boron (B) and nitrogen (N) atoms alternatingly occupy the hexagonal lattice nodes. In the bulk form, hBN was synthesized by Nagashima et al. in 1995 [31]. A decade later, the twodimensional form of $\mathrm{hBN}$ was obtained at the University of Manchester [32].

Monolayers of graphene and hBN have a very similar crystal lattice structure. Their compatibility is estimated to be $98.5 \%$ [23]. In a graphene/hBN composite, a homogeneous distribution of charge on the graphene surface is observed. This result is radically different from the data obtained for graphene/ $\mathrm{SiO}_{2}$ [33]. In addition, hBN monolayers exhibit a high temperature stability, a low dielectric constant $(\varepsilon=3-4)$, and a high thermal conductivity [34]. The band gap of hBN is about $5.9 \mathrm{eV}$ [35]. Furthermore, which is also important, hBN is nontoxic.

It is worth noting that graphene on a hBN substrate was used to fabricate transistor devices with high mobility [35], with the help of which the quantum Hall effect was observed. A heterojunction with two graphene layers [30] and superlattice structures [36-38] were also constructed. The graphene/hBN heterojunction devices allowed for the detection of the Hofstadter's butterfly phenomenon $[39,40]$. In both layer and bulk form, hBN has a large bandgap energy, which makes it an insulator $[13,41]$. Therefore, for a long time this material was not associated with superconductivity. The situation changed when it was suggested that the intercalation of lithium in hBN induces a transition to the metallic state [42]. Quasi-two-dimensional superconducting systems are currently being intensively studied for possible applications in nanometerscale superconducting quantum interference devices [43] and quantum information technology $[44,45]$.

Currently, the most promising research seems to be the properties of the superconducting state in Li-intercalated hexagonal boron nitride bilayer ( $\mathrm{Li}-\mathrm{hBN})$ compounds. Based on DFT calculations, it has been shown that the critical temperature $\left(T_{\mathrm{C}}\right)$ of the superconductor-metal phase transition is about $25 \mathrm{~K}$ [41] for the Coulomb pseudopotential $\mu^{*}=0.14$ (identical to the experimental value of $\mu^{*}$ obtained for graphene [46]). The expected value of $T_{\mathrm{C}}$ is much higher than the maximum temperature that was achieved in graphene intercalated with alkali metals ( $T_{\mathrm{C}}=8.1 \mathrm{~K}$ in Ca-intercalated bilayer graphene) [5] Also, this value is higher than that of other superconducting low-dimensional structures, e.g., $T_{\mathrm{C}} \approx 20 \mathrm{~K}$ for a Li- and Na-intercalated blue phosphorene bilayer [47], $T_{\mathrm{C}} \approx 16.5 \mathrm{~K}$ for a Li-intercalated black phosphorene bilayer [48], and $T_{\mathrm{C}} \approx 10 \mathrm{~K}$ for a $\mathrm{Li}-\mathrm{MoS}_{2}$ bilayer [49]. The obtained result for $\mathrm{Li}-\mathrm{hBN}$ is explained by the relatively high value of the electronic density of states at the Fermi level and the significant contribution to the pairing interaction from the inter-layer electron-phonon coupling [41]. This is due to the formation of characteristic bonds connecting two boron atoms in the upper and lower layers of $\mathrm{hBN}$, which results from the low electronegativity of boron atoms.

From the experimental point of view, it is worth paying attention to the results of research conducted in 2019 by S. Moriyama and co-workers [50]. A superconducting state has been observed in a system consisting of non-twisted bilayer graphene (BLG) and hexagonal boron nitride layers (hBN/BLG/ $\mathrm{hBN})$. The following characteristic temperatures were obtained: $T^{\text {onset }} \approx 50 \mathrm{~K}, T^{*} \approx 30 \mathrm{~K}$, and $T_{\mathrm{BKT}}=14 \mathrm{~K}$, which correspond the onset of superconductivity $(90 \%$ of the total transition/ normal resistance), the crossover to superconductivity (50\% of the normal resistance), and the confinement of vortices, respectively.

The important question is whether the Li-hBN bilayer system yield the high critical temperature that was suggested from DFT calculations $\left(T_{\mathrm{C}}=25 \mathrm{~K}\right)$ [41]. We think that this not the case because electron-phonon interaction in $\mathrm{Li}-\mathrm{hBN}$ needs to be taken into account together with vertex corrections. This is demonstrated by the very high ratio of $\lambda \omega_{\mathrm{D}} / \varepsilon_{\mathrm{F}} \approx 0.46$, where $\lambda=1.17$ is the electron-phonon coupling constant, $\omega_{\mathrm{D}}=165.56 \mathrm{meV}$ is the Debye frequency, and $\varepsilon_{\mathrm{F}}=417.58 \mathrm{meV}$ represents the Fermi energy [41]. Thus, in the presented paper, we characterized the properties of the superconducting state in a Li-hBN bilayer in the framework of the Eliashberg formalism, which includes the vertex corrections of electron-phonon interaction [51]. We compared the results with those obtained using the classical Migdal-Eliashberg theory [52]. Note that the use of the Eliashberg formalism is associated with the high value of the electron-phonon coupling constant $\lambda$, which characterizes the superconducting state in Li-hBN [41]. Let us remind that the BCS theory gives the correct results only in the weak-coupling limit, where $\lambda<0.3$ [53,54]. The scope of applicability of the Migdal-Eliashberg theory is carefully discussed in [55].

\section{Theoretical Model}

The classical Migdal-Eliashberg (ME) formalism [52,56] represents the natural generalization of the BCS theory (the first microscopic theory of the superconducting state) [53,54]. This generalization takes into account the retardation and strong-cou- 
pling effects of the electron-phonon interaction, which are responsible for the condensation of electrons in Cooper pairs [57] As part of the Eliashberg formalism, the electron-phonon interaction is quantified by the so-called Eliashberg function $\left(\alpha^{2} F(\omega)\right)$. The form of the Eliashberg function for a specific physical system can be determined theoretically through DFT calculations [58], or experimentally using the data provided by tunnel experiments $[59,60]$. The electron correlations (the screened Coulomb interaction) are modeled parametrically defining the so-called Coulomb pseudopotential $\left(\mu^{*}\right)$ [61]. The Eliashberg function and $\mu^{*}$ are the only input parameters of the isotropic Eliashberg equations.

The classical Eliashberg equations are thoroughly discussed in the literature [62]. They allow for the self-consistent determination of the superconducting order parameter $\left(\Delta_{n}=\Delta\left(i \omega_{n}\right)\right.$ and the wave function renormalization factor $\left(Z_{n}=Z\left(i \omega_{n}\right)\right.$, with an accuracy of the second order relative to the electron-phonon coupling function $(g)$. The symbol $\omega_{n}=\pi k_{\mathrm{B}} T(2 n+1)$ defines the fermionic Matsubara frequency. In the case of the phononinduced superconducting state, the limitation of considerations to the order of $g^{2}$ is justified by the Migdal theorem [56]. The Migdal theorem applies when the ratio $\lambda \omega_{\mathrm{D}} / \varepsilon_{\mathrm{F}}$ is of the order of 0.01 . This means that the energy of the phonons is so small that the vertex corrections for the electron-phonon interaction are irrelevant.

According to DFT calculations, the value of the ratio $\lambda \omega_{\mathrm{D}} / \varepsilon_{\mathrm{F}}$ for $\mathrm{Li}-\mathrm{hBN}$ is 0.46 . This is why the superconducting state in
Li-hBN cannot be quantified in the classical Eliashberg theory. The unusually high value of the $\lambda \omega_{\mathrm{D}} / \varepsilon_{F}$ ratio for Li-hBN is related to the fact that the physical system is quasi-two-dimensional. In the case of the bulk superconductor, the width of the electron band is significantly broadened, which results in the increase of the Fermi energy $\left(\varepsilon_{\mathrm{F}}=1.63 \mathrm{eV}\right)$. In addition, the electron-phonon coupling constant decreases $(\lambda=0.66)$. As a result, $\lambda \omega_{\mathrm{D}} / \varepsilon_{\mathrm{F}}$ is only 0.07 . The calculations carried out by us within the Migdal-Eliashberg formalism prove that the superconducting state has a significantly lower critical temperature value in the bulk than in the quasi-two-dimensional system. In particular, we obtained $T_{\mathrm{C}} \in\{14.01,8.64,4.6\} \mathrm{K}$, for $\mu^{*} \in\{0.1,0.2,0.3\}$, respectively.

To realize how uncommonly high the value of $\lambda \omega_{D} / \varepsilon_{F}$ for $\mathrm{Li}-\mathrm{hBN}$ is, it is enough to note that for the $\mathrm{Li}-\mathrm{MoS}_{2}$ bilayer, we obtain $\lambda \omega_{\mathrm{D}} / \varepsilon_{\mathrm{F}}=0.15$ [49]. In bilayers of black and blue phosphorus intercalated with lithium, $\lambda \omega_{\mathrm{D}} / \varepsilon_{\mathrm{F}}$ is equal to 0.05 and 0.1 , respectively $[47,48]$. A value of $\lambda \omega_{\mathrm{D}} / \varepsilon_{\mathrm{F}}$ of 0.09 causes a noticeable modification of the properties of the superconducting state, as in the case of $\mathrm{LiC}_{6}$, where $T_{\mathrm{C}} \approx 6 \mathrm{~K}$ $[2,46,63,64]$.

Therefore, to describe the superconducting state in Li-hBN, we used the Eliashberg equations derived with an accuracy of the fourth order relative to $g$ (lowest-order vertex corrections, LOVC). These equations were derived by Freericks et al. [51] to analyze the properties of the superconducting state in lead. They take the form given in Equation 1 and Equation $2(A=1)$ :

$$
\begin{aligned}
\varphi_{n} & =\pi k_{\mathrm{B}} T \sum_{m=-M}^{M} \frac{\lambda_{n, m}-\mu_{m}^{*}}{\sqrt{\omega_{m}^{2} Z_{m}^{2}+\varphi_{m}^{2}}} \varphi_{m} \\
& -A \frac{\pi^{3}\left(k_{\mathrm{B}} T\right)^{2}}{4 \varepsilon_{\mathrm{F}}} \sum_{m=-M}^{M} \sum_{m^{\prime}=-M}^{M} \frac{\lambda_{n, m} \lambda_{n, m^{\prime}}}{\sqrt{\left(\omega_{m}^{2} Z_{m}^{2}+\varphi_{m}^{2}\right)\left(\omega_{m^{\prime}}^{2} Z_{m^{\prime}}^{2}+\varphi_{m^{\prime}}^{2}\right)\left(\omega_{-n+m+m^{\prime}}^{2} Z_{-n+m+m^{\prime}}^{2}+\varphi_{-n+m+m^{\prime}}^{2}\right)}} \\
& \times\left[\varphi_{m} \varphi_{m^{\prime}} \varphi_{-n+m+m^{\prime}}+2 \varphi_{m} \omega_{m^{\prime}} Z_{m^{\prime}} \omega_{-n+m+m^{\prime}} Z_{-n+m+m^{\prime}}-\omega_{m} Z_{m} \omega_{m^{\prime}} Z_{m^{\prime}} \varphi_{-n+m+m^{\prime}}\right],
\end{aligned}
$$

and

$$
\begin{aligned}
Z_{n} & =1+\frac{\pi k_{\mathrm{B}} T}{\omega_{n}} \sum_{m=-M}^{M} \frac{\lambda_{n, m}}{\sqrt{\omega_{m}^{2} Z_{m}^{2}+\varphi_{m}^{2}} \omega_{m} Z_{m}} \\
& -A \frac{\pi^{3}\left(k_{\mathrm{B}} T\right)^{2}}{4 \varepsilon_{\mathrm{F}} \omega_{n}} \sum_{m=-M}^{M} \sum_{m^{\prime}=-M}^{M} \frac{\lambda_{n, m^{\prime}, m^{\prime}} \lambda_{n}}{\sqrt{\left(\omega_{m}^{2} Z_{m}^{2}+\varphi_{m}^{2}\right)\left(\omega_{m^{\prime}}^{2} Z_{m^{\prime}}^{2}+\varphi_{m^{\prime}}^{2}\right)\left(\omega_{-n+m+m^{\prime}}^{2} Z_{-n+m+m^{\prime}}^{2}+\varphi_{-n+m+m^{\prime}}^{2}\right)}} \\
& \times\left[\omega_{m} Z_{m} \omega_{m^{\prime}} Z_{m^{\prime}} \omega_{-n+m+m^{\prime}} Z_{-n+m+m^{\prime}}+2 \omega_{m} Z_{m} \varphi_{m^{\prime}} \varphi_{-n+m+m^{\prime}}-\varphi_{m} \varphi_{m^{\prime}} \omega_{-n+m+m^{\prime}} Z_{-n+m+m^{\prime}}\right],
\end{aligned}
$$


while for $A=0$, we get the classic Migdal-Eliashberg equations. The order parameter is given by the formula $\Delta_{n}=\varphi_{n} / Z_{n}$ The symbol $\lambda_{n, m}$ represents the pairing kernel for the electron-phonon interactions:

$$
\lambda_{n, m}=2 \int_{0}^{\omega_{\mathrm{D}}} \mathrm{d} \omega \frac{\omega}{\omega^{2}+4 \pi^{2}\left(k_{\mathrm{B}} T\right)^{2}(n-m)^{2}} \alpha^{2} F(\omega) .
$$

The Coulomb pseudopotential function is: $\mu_{n}^{*}=\mu^{*} \theta\left(\omega_{c}-\left|\omega_{n}\right|\right)$, where $\theta(x)$ is the Heaviside function, and $\omega_{\mathrm{c}}$ represents the cutoff frequency $\left(\omega_{c}=3 \omega_{D}=496.7 \mathrm{meV}\right)$.

Freericks' equations allow one to determine the values of the order parameter and the wave function renormalization factor in a self-consistent manner, which is undoubtedly their great advantage. These are isotropic equations, which means that the self-consistent procedure does not apply to the electron momentum (k). From the physical point of view this should not be significant, because the phonon-induced superconducting state is highly isotropic [62]. The situation would of course change radically if, in addition, the strong electron correlations had to be taken into account. Eliashberg equations including vertex corrections and an explicit dependence on $\mathbf{k}$ are also given in the literature [65-67]. These equations were derived in the context of research on the superconducting state in fullerene systems $[68,69]$, in high- $T_{\mathrm{C}}$ cuprates $[70-72]$, in heavy fermion compounds [73], and in superconductors under high magnetic fields [74]. Unfortunately, due to enormous mathematical difficulties, their full self-consistent solutions are still unknown $\left(\Delta_{n, \mathbf{k}}\right.$ and $\left.\mathrm{Z}_{n, \mathbf{k}}\right)$.

Also, Freericks' equations have been recently successfully used to analyze the superconducting state with high critical temperature values in compounds such as $\mathrm{PH}_{3}\left(T_{\mathrm{C}} \approx 80 \mathrm{~K}\right), \mathrm{H}_{3} \mathrm{~S}$ $\left(T_{\mathrm{C}} \approx 200 \mathrm{~K}\right)$ [75], and $\mathrm{H}_{2} \mathrm{~S}\left(T_{\mathrm{C}} \approx 35 \mathrm{~K}\right)$ [76].

From the mathematical point of view, the Eliashberg equations are solved in a self-consistent manner taking into account the correspondingly large number of fermionic Matsubara frequencies $[77,78]$. In our considerations, we assumed that this number $(M)$ is 4000 , which ensured the appropriate convergence of solutions of the Eliashberg equations for a temperature higher or equal to $T_{0}=4 \mathrm{~K}$. Due to the lack of experimental data in the examined physical system we took into account the Coulomb pseudopotential in a range from 0.1 to 0.2 , while the value of 0.14 was already considered in [41].

\section{Results}

In Figure 1, we plotted the dependence of the order parameter on the temperature. Note that under the imaginary axis formalism, it is assumed that the physical value of the order parameter is $\Delta_{n=1}$. In the classic ME model, we obtained the following critical temperature values: $T_{\mathrm{C}}^{\mathrm{ME}} \in\{31.9,26.9,21\} \mathrm{K}$, respectively, for $\mu^{*} \in\{0.1,0.14,0.2\}$. Comparing the obtained results with the results taking into account the impact of the vertex corrections, $\left.T_{\mathrm{C}}^{\mathrm{LOVC}} \in\{19.1,15.5,11.8\} \mathrm{K}\right)$, we find that the nonadiabatic superconducting state in $\mathrm{Li}-\mathrm{hBN}$ has a much lower value of $T_{\mathrm{C}}$ than it would follow from the ME model.

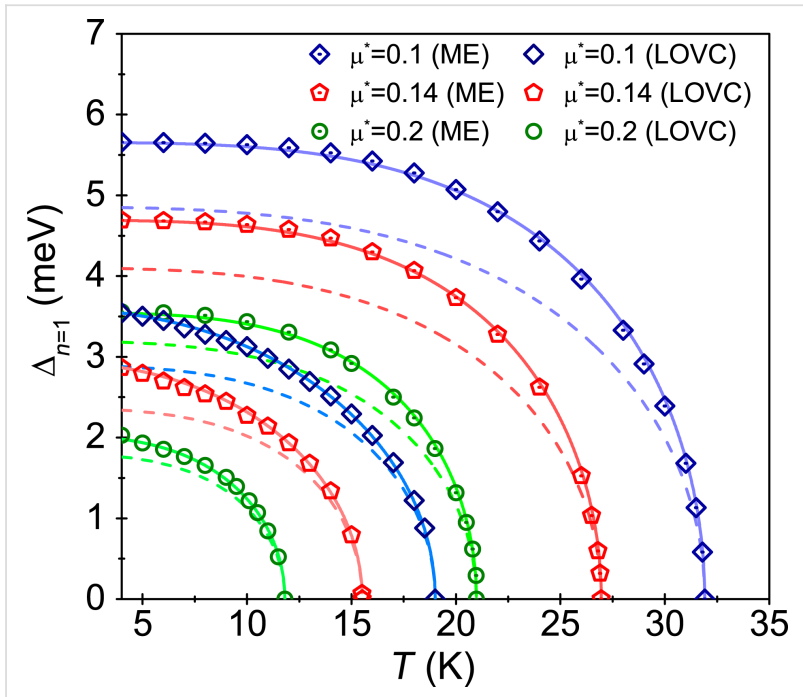

Figure 1: The order parameter as a function of the temperature. ME model - symbols with the dot, LOVC model - empty symbols, $\mu^{*} \in\{0.1,0.14,0.2\}$. The solid lines represent the parameterization of numerical results using Equation 6 . The dashed lines were obtained as part of the BCS theory (mean-field theory).

The observed lowering of the critical temperature value does not only result from the static corrections (Stat.), a good measure of which is the ratio $m=\omega_{\mathrm{D}} / \varepsilon_{\mathrm{F}}=0.4$ (Migdal parameter). It is also associated with dynamic corrections modeled by the explicit dependence of the order parameter and the wave function renormalization factor on the Matsubara frequency. Based on the results of $[67,79]$, the impact of static vertex corrections on the critical temperature can be estimated using the formula

$$
T_{\mathrm{C}}^{\mathrm{Stat} .}=\chi T_{\mathrm{C}}^{\mathrm{AD}}
$$

where $T_{\mathrm{C}}^{\mathrm{AD}}$ is the critical temperature value calculated on the basis of the Allen-Dynes formula [80]. The input from the static part of the vertex corrections has the form

$$
\chi=\frac{1}{m+1} e^{\frac{2 m-1}{2(m+1)}}
$$


A good measure of the dynamic vertex corrections is

$$
D=\frac{T_{\mathrm{C}}^{\mathrm{Stat}}-T_{\mathrm{C}}^{\mathrm{LOVC}}}{T_{\mathrm{C}}^{\mathrm{ME}}-T_{\mathrm{C}}^{\mathrm{LOVC}}} \cdot 100 \% .
$$

The results are summarized in Table 1. As one can see, the static part of the vertex corrections is responsible for $80-90 \%$ of the difference in $T_{\mathrm{C}}$.

Table 1: Critical temperature estimated from the LOVC model, from the ME model, using the Allen-Dynes formula [80], and from the analytical model including static corrections ( $\left.T_{\mathrm{C}}^{\text {Stat. }}\right)$. Additionally, the values of the $D$ parameter were given.

\begin{tabular}{llllll}
$\mu^{*}$ & $T_{\mathrm{C}}^{\mathrm{LOVC}}(\mathrm{K})$ & $T_{\mathrm{C}}^{\mathrm{ME}}(\mathrm{K})$ & $T_{\mathrm{C}}^{\mathrm{AD}}(\mathrm{K})$ & $T_{\mathrm{C}}^{\mathrm{Stat} .}(\mathrm{K})$ & $D \%$ \\
\hline 0.1 & $\mathbf{1 9 . 1}$ & 31.9 & 32.2 & $\mathbf{2 1 . 4}$ & $\mathbf{1 8}$ \\
0.14 & $\mathbf{1 5 . 5}$ & 26.9 & 26.7 & $\mathbf{1 7 . 8}$ & $\mathbf{2 0 . 2}$ \\
0.2 & $\mathbf{1 1 . 8}$ & 21 & 19.4 & $\mathbf{1 2 . 9}$ & $\mathbf{1 2}$
\end{tabular}

The numerical results obtained from the Eliashberg equations can be parameterized using the formula [81]

$$
\Delta(T)=\Delta(0) \sqrt{1-\left(T / T_{\mathrm{C}}\right)^{\Gamma}}
$$

where $\Delta(0)=\Delta\left(T_{0}\right)$. Using the LOVC model, we obtained $\Gamma \in\{2.17,2.2,2.8\}$, respectively, for $\mu^{*} \in\{0.1,0.14,0.2\}$. The exponent $\Gamma$ for the classic ME approach differs significantly in values, i.e., $\Gamma \in\{3.45,3.4,3.45\}$, respectively. The accuracy of analytical parameterization of the numerical results is presented in Figure 1 (solid lines). In addition, the results obtained under the mean-field BCS model are given by the dashed lines. In this case, $\Delta(0)=1.76 \cdot k_{\mathrm{B}} T_{\mathrm{C}}$ was adopted [53,54]. The value of the exponent $\Gamma$ for the BCS model is 3 [81].

Note the differences in the shape of the curves corresponding to the parameterization of the Eliashberg results and the BCS theory. In the case of the ME model, the differences result only from retardation and strong-coupling effects correctly taken into account in the ME formalism. These effects can be characterized by calculating the value of the ratio $r=k_{\mathrm{B}} T_{\mathrm{C}} / \omega_{\ln }$, where

$$
\omega_{\ln }=\exp \left[\frac{2}{\lambda} \int_{0}^{+\infty} \mathrm{d} \Omega \frac{\alpha^{2} F(\Omega)}{\Omega} \ln (\Omega)\right]=28.98 \mathrm{meV},
$$

is the logarithmic phonon frequency [80]. The $r$ parameter for $\mathrm{Li}-\mathrm{hBN}$ is $r^{\mathrm{ME}} \in\{0.095,0.08,0.062\}$ or $r^{\mathrm{LOVC}} \in\{0.057$, $0.046,0.035\}$, respectively, for $\mu^{*} \in\{0.1,0.14,0.2\}$. This means that the effects considered are significant even when we consider the vertex corrections for the electron-phonon interaction. Also note that retardation and strong-coupling effects for $\mathrm{Li}-\mathrm{hBN}$ are of the same order as in $\mathrm{Li}-\mathrm{MoS}_{2}$ bilayer compounds [49], Li-black phosphorene bilayers [48], and Li-blue phosphorene bilayers [47], i.e., 0.068, 0.094, and 0.099, respectively. These results were obtained for $T_{\mathrm{C}}$ determined from the Allen-Dynes formula [80] assuming $\mu^{*}=0.1$. In the BCS limit, the Eliashberg equations predict $r \rightarrow 0$.

In the LOVC theory, we take into account the vertex corrections as well as the retardation and strong-coupling effects. As a result, the differences between the Eliashberg parameterization curves and the BCS curves noticeably increase. A good measure of this effect is the value of the ratio $R_{\Delta}=2 \Delta(0) / k_{\mathrm{B}} T_{\mathrm{C}}$. For the Li-hBN system, we obtained $R_{\Delta}^{\mathrm{LOVC}} \in\{4.6,4.29,3.99\}$ and $R_{\Delta}^{\mathrm{ME}} \in\{4.12,4.04,3.9\}$. It should be emphasized that using BCS theory, a value of $R_{\Delta}=3.53$ is obtained. It is the universal constant of the model $[53,54]$. The results obtained for $\mu^{*} \in$ $\langle 0.1,0.2\rangle$ are presented in Figure 2. One can notice an interesting effect, namely, that with the increase of depairing electron correlations, the impact of vertex corrections on the ratio $R_{\Delta}$ decreases, i.e., for $\mu^{*} \approx 0.2$ the parameter $R_{\Delta}^{\mathrm{LOVC}}$ differs only slightly from $R_{\Delta}^{\mathrm{ME}}$.

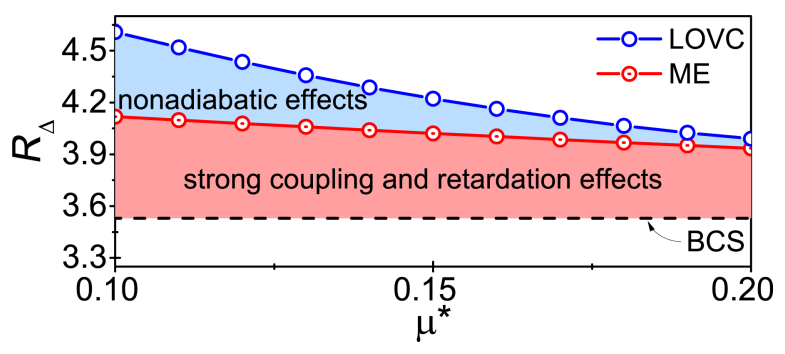

Figure 2: The values of the ratio $R_{\Delta}$ as a function of the Coulomb pseudopotential. The results obtained under the model: LOVC, ME, and BCS.

Knowing the full dependence of the order parameter on the Matsubara frequency, we determined the normalized density of states:

$$
\frac{N_{\mathrm{S}}(\omega)}{N_{\mathrm{N}}(\omega)}=\operatorname{Re}\left[\frac{|\omega-\mathrm{i} \delta|}{\sqrt{(\omega-\mathrm{i} \delta)^{2}-(\Delta(\omega))^{2}}}\right]
$$

where the pair breaking parameter $\delta$ equals $0.15 \mathrm{meV}$. We calculated the value of $\Delta(\omega)$ by continuing the function $\Delta_{n}$ on the real axis [82]. The results obtained by using the LOVC approach for $N_{\mathrm{S}}(\omega) / N_{\mathrm{N}}(\omega)$ are given in Figure $3 \mathrm{a}-\mathrm{c}$. The presented 
curves can also be determined on the basis of the data obtained using the tunneling junction. Hence, any experimental results directly relate to the predictions of the Eliashberg formalism taking into account the effect of vertex corrections. Additionally, in Figure 3d-f we plotted the form of the order parameter on the real axis $(T=4 \mathrm{~K})$. The real part of the function $\Delta(\omega)$ specifies the physical value of the order parameter, which can be calculated using the equation $\Delta(T)=\operatorname{Re}[\Delta(\omega=\Delta(T))]$ [62]. In the present case, we obtained values that differ from $\Delta_{n=1}$ by no more than $10^{-2} \%$. This result proves that the analytical continuation was correct. The imaginary part of $\Delta(\omega)$ determines the damping effects. One can see that at low frequencies, where $\operatorname{Im}[\Delta(\omega)]=0$, these effects do not occur. From the physical point of view, this means the infinite lifetime of the Cooper pairs. Above the frequency $\omega \approx 15 \mathrm{meV}$, both the real and imaginary part of the order parameter function have a complicated course. This fact results directly from the complicated shape of the Eliashberg function, which models the electron-phonon interaction in the Li-hBN system.

Let us now discuss the effect of vertex corrections on the electron band mass $\left(m_{e}\right)$. To do this, it is necessary to use the formula $m_{\mathrm{e}}^{*} / m_{\mathrm{e}}=Z_{n=1}$, where $m_{\mathrm{e}}^{*}$ is the effective electron mass.
The results obtained on the basis of the Eliashberg equations are presented in Figure 4. It is easy to see that the effective mass of the electron is almost twice as high as the electron band mass, with $m_{\mathrm{e}}^{*}$ depending very slightly on the temperature. The vertex corrections lower the value of $m_{\mathrm{e}}^{*}$ compared to the value predicted under ME formalism. If the temperature equals the critical temperature, this effect can be characterized analytically. Based on Equation 2, we obtained

$$
\begin{aligned}
Z_{n=1}= & 1+\lambda \sum_{m=-M}^{M} \operatorname{sgn}\left(\omega_{m}\right) \\
& -\lambda^{2} \frac{\pi^{2}}{4} \frac{k_{\mathrm{B}} T_{\mathrm{C}}}{\varepsilon_{\mathrm{F}}} \sum_{m, m^{\prime}=-M}^{M} \operatorname{sgn}\left(\omega_{m}\right) \operatorname{sgn}\left(\omega_{m^{\prime}}\right) \operatorname{sgn}\left(\omega_{m+m^{\prime}}\right) .
\end{aligned}
$$

Hence,

$$
Z_{n=1}^{\mathrm{LOVC}}-Z_{n=1}^{\mathrm{ME}}=-\lambda^{2}\left(\frac{\pi}{4} \frac{\omega_{\mathrm{D}}}{\varepsilon_{\mathrm{F}}}+\frac{\pi^{2}}{2} \frac{k_{\mathrm{B}} T_{\mathrm{C}}}{\varepsilon_{\mathrm{F}}}\right)
$$

where $Z_{n=1}^{\mathrm{ME}}=1+\lambda$. We see that the lowest-order vertex corrections lower the effective mass the a greater extent when $\lambda$ and $\omega_{D}$ are higher. It should be noted that in this case the critical
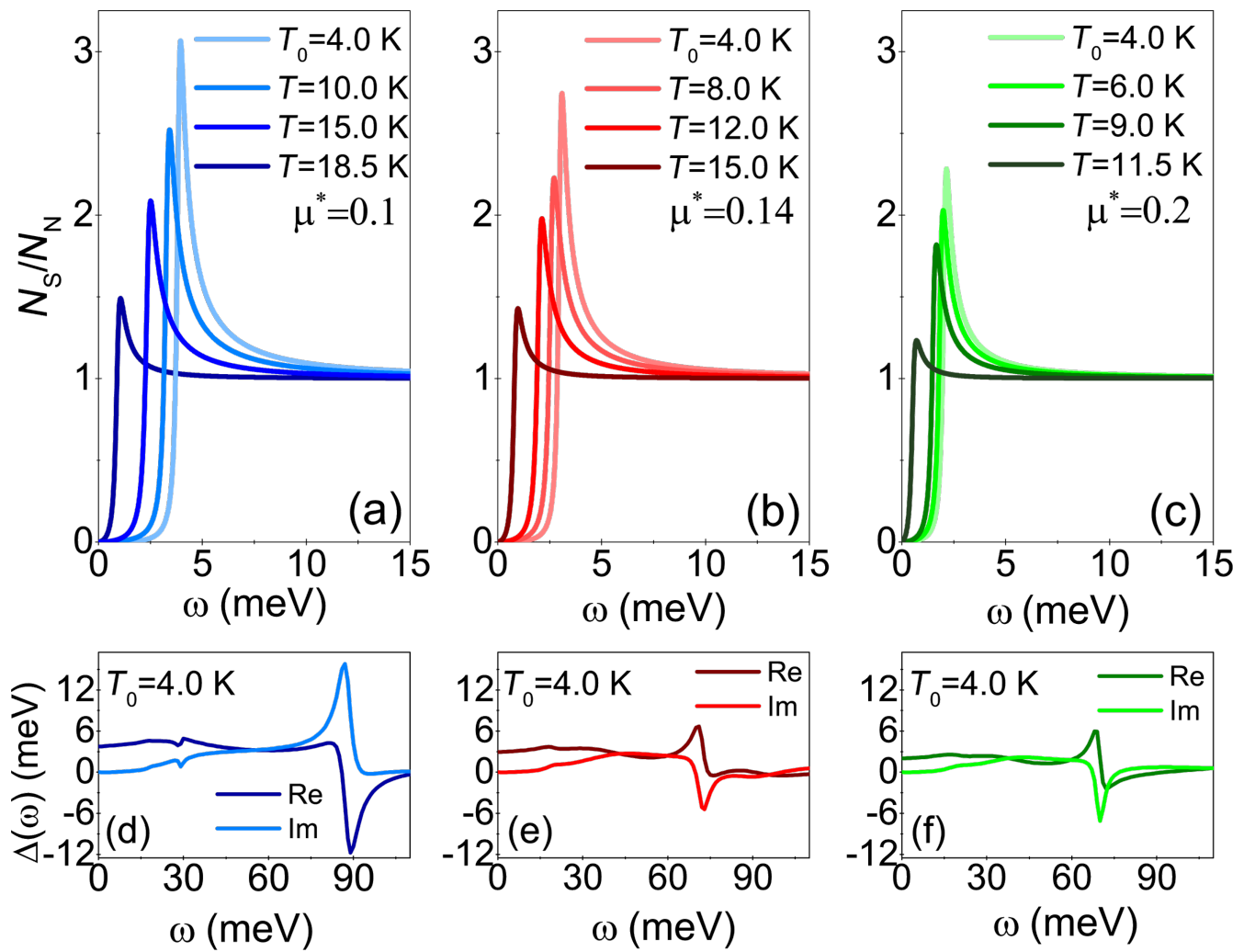

Figure 3: $(\mathrm{a}-\mathrm{c})$ Normalized density of states for the given temperatures. (d-f) The form of the order parameter on the real axis calculated for $T=4 \mathrm{~K}$. The results were obtained in the framework of the LOVC model. 
temperature also increases. The values of $Z_{n=1}^{\mathrm{LOVC}}$ and $Z_{n=1}^{\mathrm{ME}}$, calculated on the basis of Equation 9, have been marked on Figure 4 using black spheres. We obtained a good agreement between numerical and analytical results.

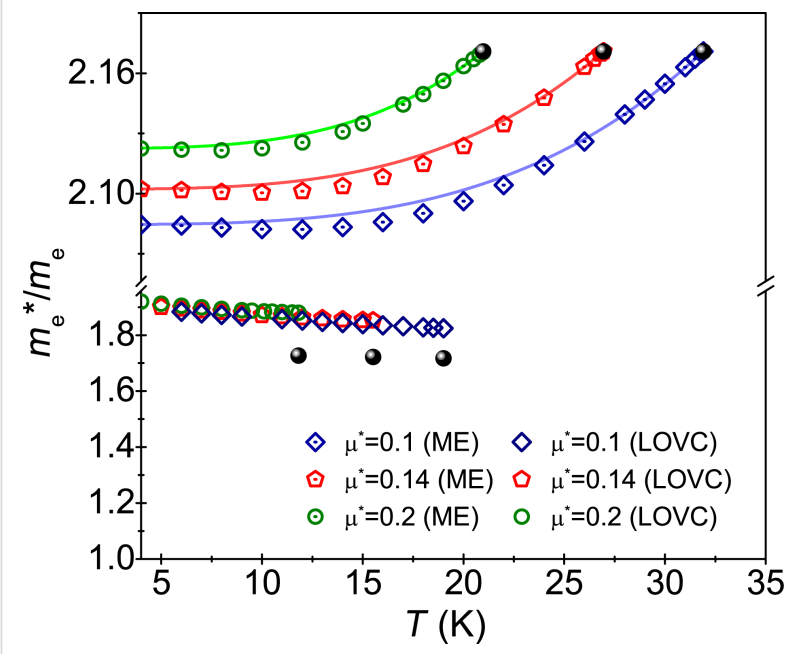

Figure 4: The ratio of the electron effective mass to the electron band mass as a function of temperature. The results were obtained in the framework of LOVC model, ME model, and Equation 9. The lines for ME results can be reproduced using the formula: $m_{\mathrm{e}}^{*} / m_{\mathrm{e}}=$ $\left[Z_{n=1}\left(T_{\mathrm{C}}\right)-Z_{n=1}(0)\right]\left(T / T_{\mathrm{C}}\right)^{\Gamma}+Z_{n=1}(0)$, where $Z_{n=1}(0)=Z_{n=1}\left(T_{0}\right)$.

\section{Discussion}

A characterization of the superconducting state in Li-hBN was carried out with the help of the Eliashberg formalism taking into account the lowest-order vertex corrections. Due to the very high value of the ratio $\lambda \omega_{\mathrm{D}} / \varepsilon_{\mathrm{F}}$, the question regarding the significance of higher-order vertex corrections arises. It turns out that no general answer can be given, as this would require the selfconsistent solution of the Eliashberg equations taking into account the vertex corrections of all orders. However, one can give arguments that support the results presented in this paper:

(1) First of all, it should be noted that the very high value of the ratio $\lambda \omega_{\mathrm{D}} / \varepsilon_{\mathrm{F}}$ does not mean that higher-order vertex corrections are equally or even more important than the lowest-order corrections. This is because the renormalization of the bare vertex amplitude $g$ has the form [66]:

$$
g \rightarrow g\left[1+\sum_{j=1}^{+\infty}\left(\lambda \omega_{\mathrm{D}} / \varepsilon_{\mathrm{F}}\right)^{j} p_{j}(\mathbf{q}, \omega)\right]
$$

where the functions $p_{j}(\mathbf{q}, \omega)$ characterize the dependence of the vertex corrections on the momentum $(\mathbf{q})$ and the frequency $(\omega)$ of the outgoing phonon. This means that not only the value of the ratio $\lambda \omega_{\mathrm{D}} / \varepsilon_{\mathrm{F}}$ is important, but also the values of all functions $p_{j}(\mathbf{q}, \omega)$.

(2) The significance of the presented results can be thoroughly understood by analyzing the normal state within the LOVC model. In this case, the self-energy $\Sigma\left(i \omega_{n}\right)$ depends only on the wave function renormalization factor, because for a temperature equal to or higher than the critical temperature, the order parameter disappears. The values of the wave function renormalization factor obtained by us are easiest to analyze taking into account Equation 9, which should be written as

$$
Z_{n=1}^{\mathrm{LOVC}}=1+\lambda-\gamma \lambda^{2}
$$

where

$$
\gamma=\frac{\left(\frac{\pi}{4} \omega_{\mathrm{D}}+\frac{\pi^{2}}{2} k_{\mathrm{B}} T_{\mathrm{C}}\right)}{\varepsilon_{\mathrm{F}}} .
$$

For the assumed values of the Coulomb pseudopotential, the parameter $\gamma$ is in the range from 0.323 to 0.331 . Therefore, for Li-hBN it is not high enough to lower $Z_{n=1}^{\mathrm{LOVC}}$ below 1 , which would indicate the loss of stability of the examined compound [83].

Regarding literature results based on models that include the lowest-order vertex corrections [67,84-86], the sign of determined parameter $\gamma$ is correct. This means that the static contribution of the vertex corrections to the normal self-energy has been qualitatively calculated in an appropriate manner. Due to the fact that Equation 9 reproduces well the numerical results (see Figure 4), the same can also be said about the self-consistent results. However, the values of the parameter $\gamma$ for the models analyzed in [85] may differ significantly, which is associated with the assumed approximations. Our result is comparable with the estimates based on $[67,85]$, where the $\gamma$ parameter is equal to 0.317 . However, it is lower than the values of $0.547,0.685$ and 1.07 that are obtained on the basis of [84-86]. On the other hand, the parameter $\gamma$ determined by us exceeds the value of $\gamma_{\mathrm{W}}=0.034$ [87], which was obtained based on the Ward identity $[85,88,89]$. Note that Ward-type identities result from the conservation of the total charge and the total spin of fermions, as a result of which one can obtain the exact relationships between the self-energy and the vertex corrections. Nevertheless, the Ward identity considered in the context of superconducting states is an equation for two functions (scalar and vector) and, thus, allows for multiple solutions. Therefore, 


$$
\begin{aligned}
& \frac{\left(k_{\mathrm{B}} T\right)^{2}}{N^{3}} \sum_{\mathbf{q}, \mathbf{q}_{1}, \mathbf{q}_{2}, \mathbf{q}_{3}} \sum_{m, m^{\prime}} K_{\mathbf{q}_{3}}^{a}\left(\omega_{m}-\omega_{m^{\prime}}\right) K_{\mathbf{q} \mathbf{q}_{1} \mathbf{q}_{2}}^{b}\left(\omega_{n}-\omega_{m}\right) \\
& \times K_{\mathbf{k}-\mathbf{q}-\mathbf{q}_{1}, \mathbf{q}_{3}}^{c}\left(\omega_{m}, \omega_{m^{\prime}}\right) K_{\mathbf{k q} \mathbf{q}_{1} \mathbf{q}_{2}}^{d}\left(\omega_{n}\right) \frac{\varphi_{\mathbf{k}-\mathbf{q}-\mathbf{q}_{1}-\mathbf{q}_{3}}\left(i \omega_{m^{\prime}}\right)}{D_{\mathbf{k}-\mathbf{q}_{-}-\mathbf{q}_{1}}\left(\omega_{m}\right) D_{\mathbf{k}-\mathbf{q}-\mathbf{q}_{1}-\mathbf{q}_{3}}\left(\omega_{m^{\prime}}\right)} .
\end{aligned}
$$

$$
\begin{aligned}
& \frac{\left(k_{\mathrm{B}} T\right)^{2}}{N^{3}} \sum_{\mathbf{q}, \mathbf{q}_{1}, \mathbf{q}_{2}, \mathbf{q}_{3}} \sum_{m} \frac{\omega_{m}}{\omega_{n}} K_{\mathbf{q q}_{1} \mathbf{q}_{2}}^{b}\left(\omega_{n}-\omega_{m}\right) K_{\mathbf{k q q}_{1} \mathbf{q}_{2}}^{e}\left(\omega_{n}\right) \times \\
& {\left[K_{\mathbf{k}-\mathbf{q}-\mathbf{q}_{1}, \mathbf{q}_{3}}^{f}\left(\omega_{m}\right)+\sum_{m^{\prime}} \frac{\omega_{m^{\prime}}}{\omega_{m}} K_{\mathbf{q}_{3}}^{a}\left(\omega_{m}-\omega_{m^{\prime}}\right) K_{\mathbf{k}-\mathbf{q}-\mathbf{q}_{1}, \mathbf{q}_{3}}^{g}\left(\omega_{m}\right)\right] \frac{Z_{\mathbf{k}-\mathbf{q}-\mathbf{q}_{1}-\mathbf{q}_{3}}\left(i \omega_{m^{\prime}}\right)}{D_{\mathbf{k}-\mathbf{q}-\mathbf{q}_{1}}\left(\omega_{m}\right) D_{\mathbf{k}-\mathbf{q}-\mathbf{q}_{1}-\mathbf{q}_{3}}\left(\omega_{m^{\prime}}\right)} .}
\end{aligned}
$$

based on the results presented in [85,90-92] one can obtain the opposite value $\gamma=-0.9$.

To sum up, the presented LOVC model predicts a small decrease in the value of the wave function renormalization factor relative to other approaches based on the lowest-order vertex corrections. This result reduces the risk of stability loss of the examined system as a result of taking into account higher-order corrections. The estimated values of the parameter $\gamma$ are very close to the value based on $[67,85]$, and are higher than $\gamma_{W}$, with the reservations made regarding the method based on the Ward identity.

(3) It is very difficult to justify our results for the values of temperatures lower than $T_{\mathrm{C}}$, where self-consistent calculations are required for $\varphi_{n}$ and $Z_{n}$. Hence, we discuss the impact on our predictions of vertex corrections of the order of $g^{6}$ (which corresponds to $\lambda^{3}$ ). Due to the very complicated form of the considered contributions, we take into account the case $\left|T_{\mathrm{C}}-T\right| \ll T_{\mathrm{C}}$. This approach allows us to linearize the Eliashberg equations [62], which greatly simplifies the numerical analysis [77]. The contributions of the order of $g^{6}$ to the Eliashberg equations were determined by using the Green thermodynamic formalism [93]. Note that the Migdal-Eliashberg approximation is based on the replacement of the mixed Green's function

$$
F_{\mathbf{k}, \mathbf{q}, \mathbf{q}^{\prime}}\left(\mathrm{i} \omega_{n}\right)=\left\langle\left\langle\Psi_{\mathbf{k}-\mathbf{q}} \phi_{\mathbf{q}} \mid \Psi_{\mathbf{k}+\mathbf{q}^{\prime}}^{\dagger} \phi_{\mathbf{q}^{\prime}}\right\rangle\right\rangle_{\mathrm{i} \omega_{n}}
$$

by the product of the full electron Green's function $G_{\mathbf{k}}\left(\mathrm{i} \omega_{n}\right)\left(\mathrm{i} \omega_{n}\right)$ and the phonon propagator for the non-interacting phonons:

$$
\left\langle\left\langle\phi_{\mathbf{q}} \mid \phi_{\mathbf{q}^{\prime}}\right\rangle\right\rangle_{\mathrm{i} \omega_{m}}
$$

We have extended this step. In particular, we have strictly determined the equation of motion for $F_{\mathbf{k}, \mathbf{q}, \mathbf{q}^{\prime}}\left(i \omega_{n}\right)\left(i \omega_{n}\right)$, and then repeated the procedure for all new unknown functions. Finally, based on the Wick theorem [94], we closed the obtained system of equations. The contribution of the order of $g^{6}$ to the first Eliashberg equation can be written as presented in Equation 12. In the case of the equation for the wave function renormalization factor we obtained the expression in Equation 13.

The explicit expressions for the kernels are given below:

$$
K_{\mathbf{q}}^{a}\left(\omega_{n}-\omega_{m}\right)=2 g_{\mathbf{q}}^{2} \frac{\omega_{\mathbf{q}}}{\left(\omega_{n}-\omega_{m}\right)^{2}+\omega_{\mathbf{q}}^{2}},
$$

$K_{\mathbf{q q}_{1} \mathbf{q}_{2}}^{b}\left(\omega_{n}-\omega_{m}\right)=-g_{\mathbf{q}} g_{-\mathbf{q}-\mathbf{q}_{1}-\mathbf{q}_{2}} g_{\mathbf{q}_{1}} g_{\mathbf{q}_{2}} B_{\mathbf{q q}_{1} \mathbf{q}_{2}}\left(\omega_{n}-\omega_{m}\right)$,

$$
K_{\mathbf{k q}}^{c}\left(\omega_{n}, \omega_{m}\right)=\frac{\left(\omega_{n}-\omega_{m}\right)^{2}}{\omega_{n}^{2}+\varepsilon_{\mathbf{k}-\mathbf{q}}^{2}},
$$

$$
K_{\mathbf{k q q}_{1} \mathbf{q}_{2}}^{d}\left(\omega_{n}\right)=\frac{-\omega_{n}^{2}-\varepsilon_{\mathbf{k}-\mathbf{q}} \varepsilon_{\mathbf{k}-\mathbf{q}-\mathbf{q}_{1}-\mathbf{q}_{2}}}{\left(\omega_{n}^{2}+\varepsilon_{\mathbf{k}-\mathbf{q}}^{2}\right)\left(\omega_{n}^{2}+\varepsilon_{\mathbf{k}-\mathbf{q}-\mathbf{q}_{1}-\mathbf{q}_{2}}^{2}\right)},
$$

$$
K_{\mathbf{k q q}_{1} \mathbf{q}_{2}}^{e}\left(\omega_{n}\right)=\frac{\omega_{n}^{2}-\varepsilon_{\mathbf{k}-\mathbf{q}} \varepsilon_{\mathbf{k}-\mathbf{q}-\mathbf{q}_{1}-\mathbf{q}_{2}}}{\left(\omega_{n}^{2}+\varepsilon_{\mathbf{k}-\mathbf{q}}^{2}\right)\left(\omega_{n}^{2}+\varepsilon_{\mathbf{k}-\mathbf{q}-\mathbf{q}_{1}-\mathbf{q}_{2}}^{2}\right)},
$$

$$
\begin{aligned}
K_{\mathbf{k q}}^{f}\left(\omega_{n}\right) & =\frac{g_{\mathbf{q}}^{2}}{k_{\mathrm{B}} T\left(\omega_{n}^{2}+\varepsilon_{\mathbf{k}-\mathbf{q}}^{2}\right)^{2}} \\
& \times\left[\left(\omega_{n}^{2}+\varepsilon_{\mathbf{k}-\mathbf{q}}^{2}\right)\left(1+2 n_{\mathbf{q}}^{p h}\right)+2 \varepsilon_{\mathbf{k}-\mathbf{q}} \omega_{\mathbf{q}}\left(2 n_{\mathbf{k}-\mathbf{q}}^{e}-1\right)\right],
\end{aligned}
$$




$$
K_{\mathbf{k q}}^{g}\left(\omega_{n}\right)=\frac{\omega_{\mathbf{q}}^{2}\left(\omega_{n}^{2}-\varepsilon_{\mathbf{k}-\mathbf{q}}^{2}\right)}{\left(\omega_{n}^{2}+\varepsilon_{\mathbf{k}-\mathbf{q}}^{2}\right)^{2}} .
$$

In Equation 14-Equation 20, $\mathrm{g}_{\mathbf{q}}$ means the electron-phonon matrix element, $\omega_{\mathbf{q}}$ represents the phonon energy, and $\varepsilon_{\mathbf{k}}$ is the electron band energy. In addition, $n_{\mathbf{k}}^{\mathrm{e}}$ means the Fermi-Dirac function and $n_{\mathbf{q}}^{\mathrm{ph}}$ is the Bose-Einstein function. The symbol

$$
B_{\mathbf{q q}_{1} \mathbf{q}_{2}}\left(\omega_{n}-\omega_{m}\right)=\left\langle\left\langle\phi_{\mathbf{q}} \phi_{\mathbf{q}_{1}} \mid \phi_{-\mathbf{q}-\mathbf{q}_{1}-\mathbf{q}_{2}} \phi_{\mathbf{q}_{2}}\right\rangle\right\rangle_{\mathrm{i}\left(\omega_{n}-\omega_{m}\right)}
$$

represents the higher-order phonon Green's function. It was designated by us assuming no interaction between phonons. The function $D_{\mathbf{k}}\left(\omega_{m}\right)$ is given by the formula

$$
D_{\mathbf{k}}\left(\omega_{m}\right)=\left[\omega_{m} Z_{\mathbf{k}}\left(i \omega_{m}\right)\right]^{2}+\varepsilon_{\mathbf{k}}^{2} .
$$

The isotropic form of contributions of the order $\lambda^{3}$ (Equation 12 and Equation 13) was obtained by exchanging the wave vector summation by the energy integration with constant density of states, wherein some integrals can be calculated numerically. We do not give the explicit isotropic expressions, because they are very extensive. We performed numerical calculations for all considered values of the Coulomb pseudopotential. For $\mu^{*} \in\{0.1,0.14,0.2\}$, we obtained a reduction of the critical temperature $T_{\mathrm{C}}^{\mathrm{LOVC}}$, respectively, by $6.2 \%, 5.4 \%$ and $4.7 \%$. This means that the vertex corrections of the order of $\lambda^{3}$ do not significantly change the critical temperature values determined with the lowest-order vertex corrections. In our opinion, the analysis presented above clearly suggests that the critical temperature in $\mathrm{Li}-\mathrm{hBN}$ is lower than $T_{\mathrm{C}}^{\mathrm{ME}}$.

In the last paragraph of this section, we discuss a possible way to increase the value of the critical temperature in $\mathrm{Li}-\mathrm{hBN}$. We believe that this is possible. To do this, consider the form of the Eliashberg function of Li-hBN (Figure 5). It can easily be seen that the Eliashberg function consists of two clearly separated parts (similar to the functions of hydrogen compounds $[95,96]$ ). In the low-frequency range $(\omega \in\{4.59,93.29\} \mathrm{meV})$ nitrogen and boron contributions are important. In the frequency range from 145.16 to $176.13 \mathrm{meV}$, the electron-phonon interaction associated with lithium atoms dominates. These frequency ranges are separable, with the Eliashberg function taking very small values in the range from 93.29 to $145.16 \mathrm{meV}$. The above facts suggest that the composition of Li-hBN could be changed to significantly increase the values of the Eliashberg function in the range from 93.29 to $145.16 \mathrm{meV}$. Most likely by appro- priate doping of the starting compound. However, this is not a simple task and requires DFT calculations.

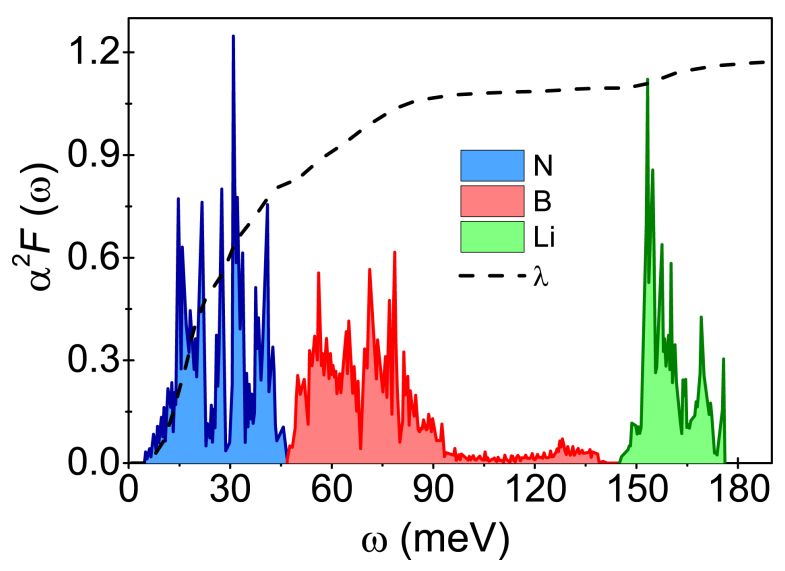

Figure 5: Eliashberg function $\alpha^{2} F(\omega)$ and electron-phonon coupling function $\lambda\left(\omega^{\prime}\right)=2 \int_{0}^{\omega^{\prime}} d \omega \alpha^{2} F(\omega) / \omega$ for Li-hBN. The results were obtained in [41]. The figure also indicates the contributions from nitrogen, boron and lithium, $\lambda^{\mathrm{N}}=0.82, \lambda^{\mathrm{B}}=0.25$, and $\lambda^{\mathrm{Li}}=0.1$, where $\lambda^{N}+\lambda^{B}+\lambda^{L i}=1.17$.

Also striking is the possibility of substitution (at least partially) of lithium by hydrogen or of boron and nitrogen by heavier elements. In the first case, the increase in critical temperature could be associated with an increase of the Debye frequency $\left(T_{\mathrm{C}} \sim \omega_{\mathrm{D}}\right.$ and $\omega_{\mathrm{D}} \sim 1 / \sqrt{M}$, while the hydrogen nucleus has a lower mass than the lithium nucleus). In the second case, the increase in $T_{\mathrm{C}}$ could result from the increase in the electron-phonon coupling constant $\left(T_{\mathrm{C}} \sim \exp (-1 / \lambda)\right.$. Contributions from heavier elements in the Eliashberg function are in the low-frequency range and are potentially more significant for $\lambda$. To find out, note that the electron-phonon coupling constant is defined by $\lambda=2 \int_{0}^{\omega_{\mathrm{D}}} \mathrm{d} \omega \alpha^{2} F(\omega) / \omega$.

\section{Conclusion}

The superconducting state in Li-hBN is induced by electron-phonon interaction, which is characterized by the uncommonly high value of the ratio $\lambda \omega_{\mathrm{D}} / \varepsilon_{\mathrm{F}}=0.46$. This means that the thermodynamic properties of the superconducting phase should be determined using a formalism explicitly including vertex corrections. Note that the very high value of the ratio $\lambda \omega_{\mathrm{D}} / \varepsilon_{\mathrm{F}}$ is related to the quasi-two-dimensionality of considered system [41].

We showed that nonadiabatic effects significantly lower the critical temperature $\left(T_{\mathrm{C}}^{\mathrm{LOVC}} \in\{19.1,15.5,11.8\} \mathrm{K}\right)$, compared to the results obtained in the framework of the Migdal-Eliashberg theory, $T_{\mathrm{C}}^{\mathrm{ME}} \in\{31.9,26.9,21\} \mathrm{K}$, for $\mu^{*} \in\{0.1,0.14,0.2\}$, respectively. In our opinion, there is no reason to believe that the critical temperature in $\mathrm{Li}-\mathrm{hBN}$ 
exceeds $20 \mathrm{~K}$, which certainly limits its applications. The vertex corrections of the order of $\lambda^{3}$ slightly decrease $T_{\mathrm{C}}^{\mathrm{LOVC}}$.

Note that the low values of $T_{\mathrm{C}}$ occur in principle in the whole family of systems in which a honeycomb crystal structure plays an important role [5,47-49]. This structure, although fundamental for the properties of graphene, is unfavorable regarding superconducting states. The reason for this is that the van Hove singularity in the electronic density of states is considerably distant form the Fermi level [97]. This is not the case for a square lattice, where the van Hove singularity is very close or even at the Fermi level, which means that the value of $T_{\mathrm{C}}$ can increase by one order of magnitude [98].

Finally, let us note that from the point of view of fundamental research on phonon-induced superconducting states, the Li-hBN system seems to be very interesting because of the unusually high value of the ratio $\lambda \omega_{\mathrm{D}} / \varepsilon_{\mathrm{F}}$, which is comparable to the value obtained for fullerene compounds [66,68]. Therefore, Li-hBN can be used to test the predictions of future theories that include vertex corrections in a fully self-consistent manner (both Matsubara frequencies and the electron wave vector $\mathbf{k}$ ). We are currently investigating this issue extensively. Preliminary results for the ME formalism can be found in $[99,100]$.

\section{Acknowledgements}

The authors would like to thank Nao H. Shimada, Emi Minamitani, and Satoshi Watanabe (University of Tokyo) for providing data on the Eliashberg function for Li-hBN bilayer, presented in [41], and for providing information on the electronic structure of bulk Li-hBN.

\section{ORCID ${ }^{\circledR}$ iDs}

Kamila A. Szewczyk - https://orcid.org/0000-0002-0319-8820 Izabela A. Domagalska - https://orcid.org/0000-0003-1361-1567 Artur P. Durajski - https://orcid.org/0000-0002-5446-4084

\section{Preprint}

A non-peer-reviewed version of this article has been previously published as a preprint https://arxiv.org/abs/2001.08732

\section{References}

1. Ohta, T.; Bostwick, A.; Seyller, T.; Horn, K.; Rotenberg, E. Science 2006, 313, 951-954. doi:10.1126/science.1130681

2. Profeta, G.; Calandra, M.; Mauri, F. Nat. Phys. 2012, 8, 131-134. doi:10.1038/nphys2181

3. Pešić, J.; Gajić, R.; Hingerl, K.; Belić, M. EPL 2014, 108, 67005. doi:10.1209/0295-5075/108/67005

4. Guzman, D. M.; Alyahyaei, H. M.; Jishi, R. A. 2D Mater. 2014, 1, 021005. doi:10.1088/2053-1583/1/2/021005

5. Margine, E. R.; Lambert, H.; Giustino, F. Sci. Rep. 2016, 6, 21414. doi:10.1038/srep21414
6. Wan, W.; Ge, Y.; Yang, F.; Yao, Y. EPL 2013, 104, 36001. doi:10.1209/0295-5075/104/36001

7. Gao, M.; Li, Q.-Z.; Yan, X.-W.; Wang, J. Phys. Rev. B 2017, 95, 024505. doi:10.1103/physrevb.95.024505

8. Liao, J.-H.; Zhao, Y.-C.; Zhao, Y.-J.; Xu, H.; Yang, X.-B. Phys. Chem. Chem. Phys. 2017, 19, 29237-29243. doi:10.1039/c7cp06180c

9. Liu, H.; Neal, A. T.; Zhu, Z.; Luo, Z.; Xu, X.; Tománek, D.; Ye, P. D. ACS Nano 2014, 8, 4033-4041. doi:10.1021/nn501226z

10. Ge, Y.; Wan, W.; Yang, F.; Yao, Y. New J. Phys. 2015, 17, 035008. doi:10.1088/1367-2630/17/3/035008

11. Shao, D. F.; Lu, W. J.; Lv, H. Y.; Sun, Y. P. EPL 2014, 108, 67004. doi:10.1209/0295-5075/108/67004

12. Kolesov, E. A. Beilstein J. Nanotechnol. 2019, 10, 531-539. doi:10.3762/bjnano.10.54

13. Wang, J.; Ma, F.; Sun, M. RSC Adv. 2017, 7, 16801-16822. doi:10.1039/c7ra00260b

14. Sławińska, J.; Zasada, I.; Klusek, Z. Phys. Rev. B 2010, 81, 155433. doi:10.1103/physrevb.81.155433

15. Hamilton, J. C.; Blakely, J. M. Surf. Sci. 1980, 91, 199-217. doi:10.1016/0039-6028(80)90080-1

16. Shikin, A. M.; Prudnikova, G. V.; Adamchuk, V. K.; Moresco, F.; Rieder, K.-H. Phys. Rev. B 2000, 62, 13202-13208. doi:10.1103/physrevb.62.13202

17. Rosei, R.; De Crescenzi, M.; Sette, F.; Quaresima, C.; Savoia, A.; Perfetti, P. Phys. Rev. B 1983, 28, 1161-1164. doi:10.1103/physrevb.28.1161

18. Shikin, A. M.; Farías, D.; Rieder, K. H. Europhys. Lett. 1998, 44, 44-49. doi:10.1209/epl/i1998-00432-x

19. Dedkov, Y. S.; Shikin, A. M.; Adamchuk, V. K.; Molodtsov, S. L.; Laubschat, C.; Bauer, A.; Kaindl, G. Phys. Rev. B 2001, 64, 035405. doi:10.1103/physrevb.64.035405

20. Brugger, T.; Günther, S.; Wang, B.; Dil, J. H.; Bocquet, M.-L.; Osterwalder, J.; Wintterlin, J.; Greber, T. Phys. Rev. B 2009, 79, 045407. doi:10.1103/physrevb.79.045407

21. Moritz, W.; Wang, B.; Bocquet, M.-L.; Brugger, T.; Greber, T.; Wintterlin, J.; Günther, S. Phys. Rev. Lett. 2010, 104, 136102. doi:10.1103/physrevlett.104.136102

22. Land, T. A.; Michely, T.; Behm, R. J.; Hemminger, J. C.; Comsa, G. Surf. Sci. 1992, 264, 261-270. doi:10.1016/0039-6028(92)90183-7

23. Starr, D. E.; Pazhetnov, E. M.; Stadnichenko, A. I.; Boronin, A. I.; Shaikhutdinov, S. K. Surf. Sci. 2006, 600, 2688-2695. doi:10.1016/j.susc.2006.04.035

24. Forbeaux, I.; Themlin, J.-M.; Debever, J.-M. Phys. Rev. B 1998, 58 , 16396-16406. doi:10.1103/physrevb.58.16396

25. Mendes-de-Sa, T. G.; Goncalves, A. M. B.; Matos, M. J. S.; Coelho, P. M.; Magalhaes-Paniago, R.; Lacerda, R. G. Nanotechnology 2012, 23, 475602. doi:10.1088/0957-4484/23/47/475602

26. Hass, J.; Varchon, F.; Millán-Otoya, J. E.; Sprinkle, M.; Sharma, N.; de Heer, W. A.; Berger, C.; First, P. N.; Magaud, L.; Conrad, E. H. Phys. Rev. Lett. 2008, 100, 125504. doi:10.1103/physrevlett.100.125504

27. Chen, J.-H.; Jang, C.; Xiao, S.; Ishigami, M.; Fuhrer, M. S. Nat. Nanotechnol. 2008, 3, 206-209. doi:10.1038/nnano.2008.58

28. Lee, D. S.; Riedl, C.; Krauss, B.; von Klitzing, K.; Starke, U.; Smet, J. H. Nano Lett. 2008, 8, 4320-4325. doi:10.1021/nl802156w

29. Geringer, V.; Liebmann, M.; Echtermeyer, T.; Runte, S.; Schmidt, M.; Rückamp, R.; Lemme, M. C.; Morgenstern, M. Phys. Rev. Lett. 2009, 102, 076102. doi:10.1103/physrevlett.102.076102 
30. Ponomarenko, L. A.; Geim, A. K.; Zhukov, A. A.; Jalil, R.; Morozov, S. V.; Novoselov, K. S.; Grigorieva, I. V.; Hill, E. H.; Cheianov, V. V.; Fal'ko, V. I.; Watanabe, K.; Taniguchi, T.; Gorbachev, R. V. Nat. Phys. 2011, 7, 958-961. doi:10.1038/nphys2114

31. Nagashima, A.; Tejima, N.; Gamou, Y.; Kawai, T.; Oshima, C. Phys. Rev. Lett. 1995, 75, 3918-3921. doi:10.1103/physrevlett.75.3918

32. Novoselov, K. S.; Jiang, D.; Schedin, F.; Booth, T. J.; Khotkevich, V. V.; Morozov, S. V.; Geim, A. K. Proc. Natl. Acad. Sci. U. S. A. 2005, 102, 10451-10453. doi:10.1073/pnas.0502848102

33. Decker, R.; Wang, Y.; Brar, V. W.; Regan, W.; Tsai, H.-Z.; Wu, Q.; Gannett, W.; Zettl, A.; Crommie, M. F. Nano Lett. 2011, 11, 2291-2295. doi:10.1021/nl2005115

34. Giovannetti, G.; Khomyakov, P. A.; Brocks, G.; Kelly, P. J.; van den Brink, J. Phys. Rev. B 2007, 76, 073103. doi:10.1103/physrevb.76.073103

35. Dean, C. R.; Young, A. F.; Meric, I.; Lee, C.; Wang, L.; Sorgenfrei, S.; Watanabe, K.; Taniguchi, T.; Kim, P.; Shepard, K. L.; Hone, J. Nat. Nanotechnol. 2010, 5, 722-726. doi:10.1038/nnano.2010.172

36. Britnell, L.; Gorbachev, R. V.; Jalil, R.; Belle, B. D.; Schedin, F.; Mishchenko, A.; Georgiou, T.; Katsnelson, M. I.; Eaves, L.; Morozov, S. V.; Peres, N. M. R.; Leist, J.; Geim, A. K.; Novoselov, K. S.; Ponomarenko, L. A. Science 2012, 335, 947-950. doi:10.1126/science. 1218461

37. Haigh, S. J.; Gholinia, A.; Jalil, R.; Romani, S.; Britnell, L.; Elias, D. C.; Novoselov, K. S.; Ponomarenko, L. A.; Geim, A. K.; Gorbachev, R. Nat. Mater. 2012, 11, 764-767. doi:10.1038/nmat3386

38. Dean, C.; Young, A. F.; Wang, L.; Meric, I.; Lee, G.-H.; Watanabe, K.; Taniguchi, T.; Shepard, K.; Kim, P.; Hone, J. Solid State Commun. 2012, 152, 1275-1282. doi:10.1016/j.ssc.2012.04.021

39. Ponomarenko, L. A.; Gorbachev, R. V.; Yu, G. L.; Elias, D. C.; Jalil, R.; Patel, A. A.; Mishchenko, A.; Mayorov, A. S.; Woods, C. R.; Wallbank, J. R.; Mucha-Kruczynski, M.; Piot, B. A.; Potemski, M.; Grigorieva, I. V.; Novoselov, K. S.; Guinea, F.; Fal'ko, V. I.; Geim, A. K. Nature 2013, 497, 594-597. doi:10.1038/nature12187

40. Dean, C. R.; Wang, L.; Maher, P.; Forsythe, C.; Ghahari, F.; Gao, Y.; Katoch, J.; Ishigami, M.; Moon, P.; Koshino, M.; Taniguchi, T.; Watanabe, K.; Shepard, K. L.; Hone, J.; Kim, P. Nature 2013, 497, 598-602. doi:10.1038/nature12186

41. Shimada, N. H.; Minamitani, E.; Watanabe, S. Appl. Phys. Express 2017, 10, 093101. doi:10.7567/apex.10.093101

42. Altintas, B.; Parlak, C.; Bozkurt, C.; Eryiğit, R. Eur. Phys. J. B 2011, 79, 301-312. doi:10.1140/epjb/e2010-10660-4

43. Fatemi, V.; Wu, S.; Cao, Y.; Bretheau, L.; Gibson, Q. D.; Watanabe, K.; Taniguchi, T.; Cava, R. J.; Jarillo-Herrero, P. Science 2018, 362, 926-929. doi:10.1126/science.aar4642

44. Fagaly, R. L. Rev. Sci. Instrum. 2006, 77, 101101. doi:10.1063/1.2354545

45. Komatsu, K.; Morita, Y.; Watanabe, E.; Tsuya, D.; Watanabe, K.; Taniguchi, T.; Moriyama, S. Sci. Adv. 2018, 4, eaaq0194. doi:10.1126/sciadv.aaq0194

46. Ludbrook, B. M.; Levy, G.; Nigge, P.; Zonno, M.; Schneider, M.; Dvorak, D. J.; Veenstra, C. N.; Zhdanovich, S.; Wong, D.; Dosanjh, P.; Straßer, C.; Stöhr, A.; Forti, S.; Ast, C. R.; Starke, U.; Damascelli, A. Proc. Natl. Acad. Sci. U. S. A. 2015, 112, 11795-11799. doi:10.1073/pnas.1510435112

47. Zhang, J.-J.; Dong, S. 2D Mater. 2016, 3, 035006. doi:10.1088/2053-1583/3/3/035006
48. Huang, G. Q.; Xing, Z. W.; Xing, D. Y. Appl. Phys. Lett. 2015, 106, 113107. doi: $10.1063 / 1.4916100$

49. Huang, G. Q.; Xing, Z. W.; Xing, D. Y. Phys. Rev. B 2016, 93, 104511. doi:10.1103/physrevb.93.104511

50. Moriyama, S.; Morita, Y.; Komatsu, K.; Endo, K.; Iwasaki, T.; Nakaharai, S.; Noguchi, Y.; Wakayama, Y.; Watanabe, E.; Tsuya, D.; Watanabe, K.; Taniguchi, T. arXiv 2019, No. 1901.09356.

51. Freericks, J. K.; Nicol, E. J.; Liu, A. Y.; Quong, A. A. Phys. Rev. B 1997, 55, 11651-11658. doi:10.1103/physrevb.55.11651

52. Eliashberg, G. M. Sov. Phys. - JETP 1960, 11, 696.

53. Bardeen, J.; Cooper, L. N.; Schrieffer, J. R. Phys. Rev. 1957, 106, 162-164. doi:10.1103/physrev.106.162

54. Bardeen, J.; Cooper, L. N.; Schrieffer, J. R. Phys. Rev. 1957, 108, 1175-1204. doi:10.1103/physrev.108.1175

55. Chubukov, A. V.; Abanov, A.; Esterlis, I.; Kivelson, S. A. Ann. Phys. (Amsterdam, Neth.) 2020, 417, 168190. doi:10.1016/j.aop.2020.168190

56. Migdal, A. B. Sov. Phys. - JETP 1958, 34, 996.

57. Cooper, L. N. Phys. Rev. 1956, 104, 1189-1190. doi:10.1103/physrev.104.1189

58. Giustino, F. Rev. Mod. Phys. 2017, 89, 015003. doi:10.1103/revmodphys.89.015003

59. McMillan, W. L.; Rowell, J. M. Phys. Rev. Lett. 1965, 14, 108-112. doi:10.1103/physrevlett.14.108

60. Yanson, I. K. Sov. Phys. - JETP 1974, 39, 506.

61. Morel, P.; Anderson, P. W. Phys. Rev. 1962, 125, 1263-1271. doi:10.1103/physrev.125.1263

62. Carbotte, J. P. Rev. Mod. Phys. 1990, 62, 1027-1157. doi:10.1103/revmodphys.62.1027

63. Zheng, J.-J.; Margine, E. R. Phys. Rev. B 2016, 94, 064509. doi:10.1103/physrevb.94.064509

64. Szczśniak, D.; Szczśniak, R. Phys. Rev. B 2019, 99, 224512. doi:10.1103/physrevb.99.224512

65. Grimaldi, C.; Pietronero, L.; Strässler, S. Phys. Rev. Lett. 1995, 75, 1158-1161. doi:10.1103/physrevlett.75.1158

66. Pietronero, L.; Strässler, S.; Grimaldi, C. Phys. Rev. B 1995, 52, 10516-10529. doi:10.1103/physrevb.52.10516

67. Grimaldi, C.; Pietronero, L.; Strässler, S. Phys. Rev. B 1995, 52, 10530-10546. doi:10.1103/physrevb.52.10530

68. Pietronero, L. Europhys. Lett. 1992, 17, 365-371. doi:10.1209/0295-5075/17/4/015

69. Pickett, W. E.; Poirier, D. M. Solid State Phys. 1994, 48, 225-347. doi:10.1016/s0081-1947(08)60580-9

70. Uemura, Y. J.; Le, L. P.; Luke, G. M.; Sternlieb, B. J.; Wu, W. D.; Brewer, J. H.; Riseman, T. M.; Seaman, C. L.; Maple, M. B.; Ishikawa, M.; Hinks, D. G.; Jorgensen, J. D.; Saito, G.; Yamochi, H. Phys. Rev. Lett. 1991, 66, 2665-2668. doi:10.1103/physrevlett.66.2665

71. Uemura, Y. J.; Le, L. P.; Luke, G. M.; Sternlieb, B. J.; Wu, W. D.; Brewer, J. H.; Riseman, T. M.; Seaman, C. L.; Maple, M. B.; Ishikawa, M.; Hinks, D. G.; Jorgensen, J. D.; Saito, G.; Yamochi, H. Phys. Rev. Lett. 1992, 68, 2712. doi:10.1103/physrevlett.68.2712

72. d'Ambrumenil, N. Nature 1991, 352, 472-473. doi:10.1038/352472a0

73. Wojciechowski, R. J. On the validity of the Migdal's theorem in the heavy fermion systems. International Centre For Theoretical Physics, Trieste; International Atomic Energy Agency, 1996; Vol. 28.

74. Goto, H.; Natsume, Y. Phys. B (Amsterdam, Neth.) 1996, 216 , 281-284. doi:10.1016/0921-4526(95)00493-9

75. Durajski, A. P. Sci. Rep. 2016, 6, 38570. doi:10.1038/srep38570 
76. Kostrzewa, M.; Szczęśniak, R.; Kalaga, J. K.; Wrona, I. A. Sci. Rep. 2018, 8, 11957. doi:10.1038/s41598-018-30391-z

77. Szczęśniak, R. Acta Phys. Pol., A 2006, 109, 179-186. doi:10.12693/aphyspola.109.179

78. Wiendlocha, B.; Szczśniak, R.; Durajski, A. P.; Muras, M. Phys. Rev. B 2016, 94, 134517. doi:10.1103/physrevb.94.134517

79. Combescot, R. Phys. Rev. B 1990, 42, 7810-7824. doi: $10.1103 /$ physrevb.42.7810

80. Allen, P. B.; Dynes, R. C. Phys. Rev. B 1975, 12, 905-922. doi:10.1103/physrevb.12.905

81. Eschrig, H. Theory of Superconductivity: A Primer; IFW: Dresden, Germany, 2001.

82. Beach, K. S. D.; Gooding, R. J.; Marsiglio, F. Phys. Rev. B 2000, 61, 5147-5157. doi:10.1103/physrevb.61.5147

83. Dolgov, O. V.; Losyakov, V. V. Phys. Lett. A 1994, 190, 189-190. doi:10.1016/0375-9601(94)90076-0

84. Cai, J.; Lei, X. L.; Xie, L. M. Phys. Rev. B 1989, 39, 11618-11623. doi:10.1103/physrevb.39.11618

85. Danylenko, O. V.; Dolgov, O. V. Phys. Rev. B 2001, 63, 094506. doi:10.1103/physrevb.63.094506

86. Kostur, V. N.; Mitrović, B. Phys. Rev. B 1994, 50, 12774-12787. doi:10.1103/physrevb.50.12774

87. Cosenza, F.; De Cesare, L.; Fusco Girard, M. Phys. Rev. B 1999, 59, 3349-3352. doi:10.1103/physrevb.59.3349

88. Ward, J. C. Phys. Rev. 1950, 78, 182. doi:10.1103/physrev.78.182

89. Engelsberg, S.; Schrieffer, J. R. Phys. Rev. 1963, 131, 993-1008. doi:10.1103/physrev.131.993

90. Takada, Y. J. Phys. Chem. Solids 1993, 54, 1779-1788. doi:10.1016/0022-3697(93)90290-8

91. Takada, Y. J. Supercond. 1995, 8, 429-432. doi:10.1007/bf00722822

92. Takada, Y.; Higuchi, T. Phys. Rev. B 1995, 52, 12720-12735. doi:10.1103/physrevb.52.12720

93. Elk, K.; Gasser, W. Die Methode der Greenschen Funktionen in der Festkörperphysik; Akademie-Verlag, 1979.

94. Fetter, A. L.; Walecka, J. D. Quantum theory of many-particle systems; McGraw-Hill Book Compan, 1971.

95. Li, Y.; Hao, J.; Liu, H.; Li, Y.; Ma, Y. J. Chem. Phys. 2014, 140, 174712. doi:10.1063/1.4874158

96. Duan, D.; Liu, Y.; Tian, F.; Li, D.; Huang, X.; Zhao, Z.; Yu, H.; Liu, B.; Tian, W.; Cui, T. Sci. Rep. 2015, 4, 6968. doi:10.1038/srep06968

97. Van Hove, L. Phys. Rev. 1953, 89, 1189-1193. doi:10.1103/physrev.89.1189

98. Szczęśniak, R. PLoS One 2012, 7, e31873. doi:10.1371/journal.pone.0031873

99. Szewczyk, K. A.; Szczśniak, R.; Szczśniak, D. Ann. Phys. (Berlin, Ger.) 2018, 530, 1800139. doi:10.1002/andp.201800139

100.Szewczyk, K. A.; Jarosik, M. W.; Durajski, A.; Szczśniak, R. arXiv 2019, No. 1904.05642.

\section{License and Terms}

This is an Open Access article under the terms of the Creative Commons Attribution License (http://creativecommons.org/licenses/by/4.0). Please note that the reuse, redistribution and reproduction in particular requires that the authors and source are credited.

The license is subject to the Beilstein Journal of Nanotechnology terms and conditions: (https://www.beilstein-journals.org/bjnano)

The definitive version of this article is the electronic one which can be found at: doi:10.3762/bjnano.11.102 